\title{
Parameter Preserving \\ Data Type Specifications
}

\author{
Peter Padawitz
}

Universitat Passau

Fakultat far Informatik

Postfach 2540

D-8390 Passau

F.R.G.

\begin{abstract}
Term rewriting methods are used for solving the persistency problem of parameterized data type specifications. Such a specification is called persistent if the parameter part of its algebraic semantics agrees with the semantics of the parameter specification. Since persistency mostly cannot be guaranteed for the whole equational variety of the parameter specification, the persistency criteria developed here mainly concern classes of parameter algebras with "built-in" logic.
\end{abstract}

\section{Persistency, extensions and inductive theories}

Starting from a many-sorted signature $\langle S, O P\rangle$ with sorts $S$ and operation symbols $O P$ an algebraic specification in this sense of $A D J / 1 /$ is given by a triple $S P E C=$ $\langle S, O P, E\rangle$ where $E$ is a set of equations between OP-terms. Algebras with signature $\langle S, O P\rangle$ which satisfy $E$ are called SPEC-algebras. For reasons discussed extensively in the literature (e.g. in $/ 1 /)$ the isomorphism class of initial SPEC-algebras plays a dominant role.

A parameterized specification PAR is a pair of two specifications PSPEC and SPEC where the parameter PSPEC is part of the target SPEC. The role of initial algebras is taken over by a class of target algebras each of which is "freely generated" over some algebra in a given class $\mathrm{K}$ of parameter algebras (cf. $/ 2 /$ ).

Such a class of target algebras is called a parameterized data type. /10/ deals with the proof-theoretical characterization of the equational variety of parameterized data types. This variety turned out to be a certain "inductive" theory of the target specification.

In many cases this characterization works only if PAR is persistent, i,e. if each algebra in the corresponding data type "preserves" the parameter algebra where it is "freely generated" upon. Persistency is also a sufficient criterion for the "passing compatibility" of PAR with actual parameter specifications (cf. ADJ /3/). So this paper is devoted to decidable and powerful criteria for persistency. The first step towards such conditions is the decomposition of PAR into a "base" specification BPAR and the remaining operations \& equations of PAR. BPAR is supposed to contain those operations \& equations of PAR that are necessary for the "construction" of data. Following this strategy it 
is mostly simple to show that BPAR is persistent. Then PAR is persistent, too, if BPAR is a conservative extension of PAR which means that the "base" part of the data type specified by PAR agrees with the data type specified by BPAR.

The extension property is separated into two parts: completeness and consistency. So the tools for solving the persistency problem are the criteria for persistency of BPAR given by Theorem 2.12 and the completeness and consistency conditions of Thms. $3.4 / 4.7$ and $3.5 / 5.14$, respectively. They involve normalization and confluence properties of term reductions and are tailor-made for parameter algebras with "built-in" logic where the proof-theoretical characterization of parameterized data types given in $110 /$, section 3 , is based upon, too.

Besides well-known notions in term rewriting theory like "confluence" and "critical pair" we use some recently introduced ones like "coherence" ( $\mathrm{cf}$. /8/), "contextual reductions" (cf. /12/) and "recursive critical pairs" (cf. /11/). They should support the reader's intuition, although their definitions sometimes deviate from their meaning in the cited papers. Moreover, the corresponding results presented here are different from those given there.

The paper is organized as follows: Section 2 contains basic definitions and proof-theoretical characterizations of conservative extensions (2.9) and persistency (2.12). In section 3 general completeness and consistency theorems $(3.4 / 5)$ are given that refer to term reductions. Sections 4 and 5 focus on parameter algebras with "built-in" logic and adapt the notions of section 3 to this case. Decidable criteria for the crucial confluence criteria of Thm 3.5 are developed in section 5 that culminates in the Critical Pair Thm. 5.12. The main results of sections 4 and 5 are summarized by Completeness Thm. 4.8, Consistency Thm. 5.14 and Persistency Thm. 5.16.

Former versions of these results are part of the author's Ph.D.thesis $19 /$. id, inc and nat denote identity, inclusion and natural mappings, respectively. The first occurrences of notions used throughout the whole paper are printed in boldface.

\section{The syntax and semantics of parameterized specifications}

Let $S I G=\langle S, O P\rangle$ be a many-sorted signature with a set $S$ of sorts and an $\left(S^{*} \times S\right)$-sorted set $O P$ of operation symbols. If $\sigma \& O P_{W, S}$, then $\operatorname{arity}(\sigma)=W$, $\operatorname{sort}(\sigma)=s$, and we often write $\sigma: w \rightarrow s$. If $w=\varepsilon$ (empty word), $\sigma$ is called a constant. I(SIG) denotes the free S-sorted algebra of oP-terms over a fixed infinite $S$-sorted set $X$ of variables. If $t, t^{\prime}=T(S I G)$ and $x \in x$, then $t\left[t^{\prime} / x\right]$ is $t$ with $x$ replaced by $t$ '.

For every $S$-sorted set $A$ and all $s 1, \ldots, s n \leq S, A_{S} 1 \ldots$ sn $:=A_{S} 1 \times \ldots \times A_{S n}$. Let $w$ $\leq S^{*}, s<S, \sigma \in O P_{W, S}$ and $t \in T(S I G)_{W}$. Then $\operatorname{root}(\sigma t)=\sigma, \arg (\sigma t)=t$, sort $(\sigma t)=s$, and op $(\sigma t)$ resp. var $(\sigma t)$ denote the set of operation symbols resp. variables of $\sigma t$. Size( $t)$ is the number of operation symbol occurrences in $t$. A SIG-equation $I=r$ is a pair of SIG-terms $I$ and $r$ with sort(l) $=\operatorname{sort}(r)$. Let $A$ be a SIG-algebra. $Z(A)$ denotes the $S$-sorted set of functions from $X$ to $A$. The unique homomorphic extension of $f \in Z(A)$ to $T(S I G)$ is also written $f$. If $f \in Z(T(S I G))$, $t=T(S I G)$ and $x \in X$, then $f[t / x] \in Z(T(S I G))$ is defined by $f[t / x](x)=t$ and $f[t / x](y)=f y$ for all $y \in X-\{x\}$.

$A$ satisfies a SIG-equation $\mathrm{l}=\mathrm{r}$ if for all $f \in Z(A) f l=f r$. (This definition extends to classes of algebras and sets of equations as usual.) 


\subsection{Definitions (specification \& semantics)}

An (equational) specification $S P E C=\langle S, O P, E\rangle$ consists of a many-sorted signature SIG $=\langle S, O P\rangle$ and a set $E$ of SIG-equations. Alg (SPEC) denotes the class of SIG-algebras that satify $E$. The free SPEC-congruence =SPEC is the smallest SIG-congruence on $T(S I G)$ that contains all pairs $\langle f l, f r\rangle$ with $1=r$ in $E$ and $f$ $Z(T(S I G))$. = SPEC is also called the free theory of SPEC.

G(SIG) denotes the free S-sorted algebra of OP-terms over the empty set. Gen(SPEC) is the class of "finitely generated" SIG-algebras that satisfy E, i.e. every $a \in A$ is the interpretation of some $t \in G(S I G)$. The inductive SPEC-congruence $=S P E C$ is given by all pairs $\left\langle t, t^{\prime}\right\rangle \in I(S I G\rangle^{2}$ such that for all $f$ a $Z(G(S I G))$ ft $=$ SPEC $\mathrm{ft}^{\prime}$. ESPEC is also called the inductive theory of SPEC. Note that the restriction of $\mathrm{BSPEC}_{\mathrm{SP}} \mathrm{G}(\mathrm{SIG})^{2}$ coincides with $=\mathrm{SPEC}$.

Two facts are well-known (of. /4/ resp. /1/):

\subsection{Theorem}

1. Alg (SPEC) satisfies $t=t$ ' iff $t=S P E C^{\prime}$ '.

2. Gen(SPEC) satisfies $t=t^{\prime}$ 'iff $t=S P E C t^{\prime}$.

\subsection{Definitions (parameterized data types)}

A parameterized specification PAR is a pair of two specifications PSPEC and SPEC. The forgetful functor from $A l g$ (SPEC) to $A l g$ (PSPEC) is denoted by UPAR, while

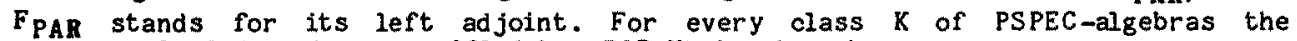
parameterized data type specified by $\langle P A R, K\rangle$ is given by

$\operatorname{PDT}(P A R, K)=\left\{F_{P A R}(A) \mid A \subset K\right\}$.

Let PSIG $=\langle$ PS, POP $\rangle$, PSPEC $=\langle P S, P O P, P E\rangle$, SPEC $=\langle S, O P, E\rangle$ and $P X=\left\{\begin{array}{lll}X & X \mid\end{array}\right.$ sort $(X) \subset P S\}$. Regarding $P X$ as constants we obtain the signature SIGX = $\langle S, O P \cup P X\rangle$ and the specification SPECX $=\langle S, O P U P X, E\rangle$. ESPECX is called the inductive theory of PAR.

Analogously to Thm. 2.2, there is the following proof-theoretical characterization of the data type specified by $\langle P A R, A l g(P S P E C)\rangle$ :

\subsection{Theorem $(/ 10 /, 1.7)$}

PDT (PAR,Alg (PSPEC)) satisfies $t=t^{\prime}$ iff $t=S P E C X t^{\prime}$.

\subsection{Definitions (persistency * extension)}

Let ID be the identity functor on $A 1 g$ (PSPEC). We recall from category theory that there is a functor transformation $\eta_{P A R}: I D \rightarrow U_{P A R} F_{P A R}$ such that for all $B E$ $A l_{g}$ (SPEC) each homomorphism $h: A \rightarrow U_{P A R}(B)$ uniquely extends to a homomorphism $h^{*}: F_{P A R}(A) \rightarrow B$ such that $U_{P A R}\left(h^{*}\right) \circ \eta_{P A R}(A)=h$.

Let $K$ be a class of PSPEC-algebras. $\langle P A R, K\rangle$ is persistent if for all $A E K$ $\eta P_{A R}(A)$ is bijective.

Let BPAR = 〈PSPEC,BSPEC > be a parameterized subspecification of PAR, i.e. BSPEC $=\langle B S, B O P, B E\rangle$ is componentwise included in $S P E C$. Let $B S I G=\langle B S, B O P\rangle$ and $E X T=$ $\langle B S P E C, S P E C\rangle$. Since $U_{P A R}=U_{B P A R} \circ U_{E X T}, \eta_{P A R}(A): A \rightarrow U_{P A R} F_{P A R}(A)$ uniquely 
extends to $\eta_{P A R}(A): F_{B P A R}(A) \rightarrow U_{E X T F P A R}(A)$ such that $U_{B P A R}\left(\eta P A R(A)^{*}\right) \circ$ $\eta_{B P A R}(A)=\eta_{P A R}(A):$

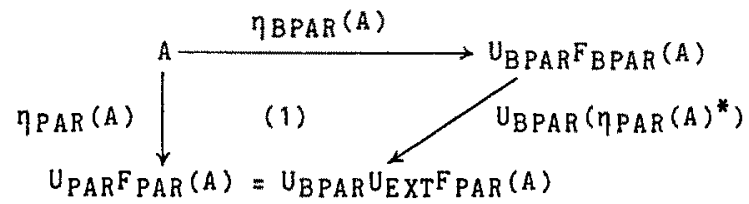

$P A R$ is complete (consistent) w.r.t. 〈BPAR, $K\rangle$ if for all $A \in K \eta_{P A R}(A)^{*}$ is surjective (injective). $P A R$ is a conservative extension of 〈BPAR, $K\rangle$ if $P A R$ is complete and consistent w.r.t. 〈BPAR,K〉.

An immediate consequence of these definitions is the following

\subsection{Decomposition Lemma for Persistency}

Let 〈BPAR, $K\rangle$ be persistent. PAR is a conservative extension of $\langle B P A R, K\rangle$ iff $\langle P A R, K\rangle$ is persistent.

\subsection{Example}

Let BOOL be a specification of Boolean aigebras, i.e. BOOL consists of a sort bool, constants true and false, operation symbols $\neg, \wedge, \vee, \Rightarrow, \Leftrightarrow$ and the Boolean algebras axioms. Moreover,

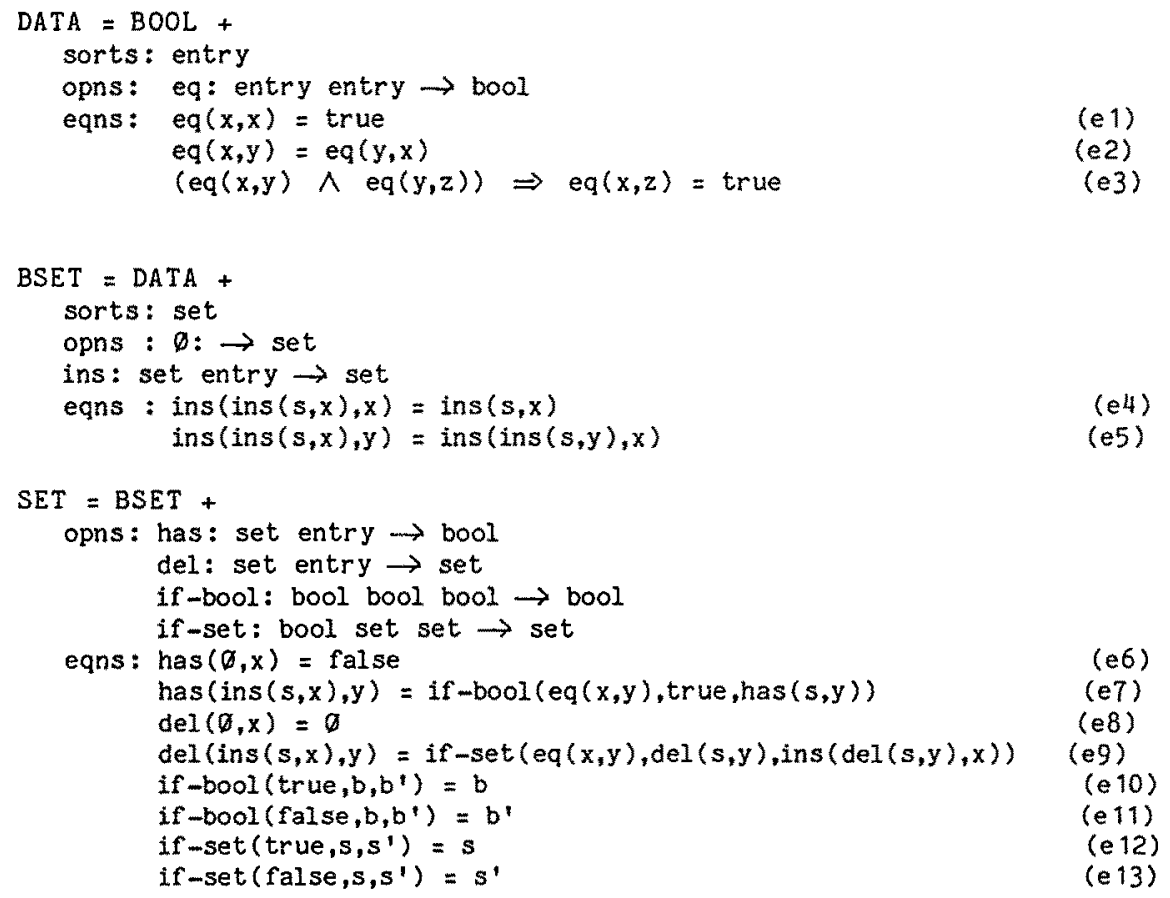


Following the strategy developed in this paper we will show that for a certain class Log of DATA-algebras, which will be given in section 4, 〈SET,LOg(DATA)〉 is persistent.

We proceed with the representation of parameterized data types by classes of initial algebras which is essential for the proof-theoretical characterization of conservative extensions (2.9).

\subsection{Definition and Theorem $(/ 10 /, 1.5)$}

Let $A$ a $A 1 g$ (PSPEC). The specification $\operatorname{SPEC}(A)=\langle S, O P \cup A, E \cup \triangle(A)\rangle$

has all operation symbols of SPEC together with all elements of $A$ as constants, while the set of equations of SPEC is extended by the equational diagram of $A$, $\triangle(A)$, that consists of all equations $\sigma(a)=\sigma_{A}(a)$ with $\sigma \in$ POP and $a \in$ $A_{\text {arity }(\sigma) \text {. }}$

$F_{\text {PAR }}(A)$ gets an (OP $\cup A$ )-algebra by interpreting each constant $a \in A$ by $\eta_{P A R}(A)(a)$. Moreover, $F_{P A R}(A)$ is an initial object in $\operatorname{Alg}(\operatorname{SPEC}(A))$.

Using the well-known quotient term algebra representation of initial algebras ( $\mathrm{cf}$. $A D J / 1 /$ ) we can formulate completeness and consistency as free theory properties:

\subsection{Theorem}

Let $\operatorname{BSIG}(A)=\langle B S, B O P \cup A\rangle$ and $\operatorname{SIG}(A)=\langle S, O P \cup A\rangle$.

1. PAR is complete w.r.t. $\langle B P A R, K\rangle$ iff for all $A \in K, s \in B S$ and $t \in G(S I G(A))_{S}$ some t' $C G(B S I G(A))$ satisfies $t=S P E C(A) t^{\prime}$.

2. PAR is consistent w.r.t. 〈BPAR, $K$ 〉 iff for all $A \in K$ and $t, t$ ' $\in G(B S I G(A))$ $t=\operatorname{SPEC}(A) t^{\prime}$ implies $t=\operatorname{BSPEC}(A) \quad t$.

Besides persistency Thms. $2.8 \approx 2.9$ provide a useful criterion for the validity of equations in parameterized data types: PDT(PAR, $K)$ satisfies a set $E^{\prime}$ of SIG-equations if $P A R$ is complete w.r.t. $\langle B P A R, K\rangle$ and $\left\langle P S P E C,\left\langle S, O P, E \cup E^{\prime}\right\rangle\right\rangle$ is consistent w.r.t. $\langle B P A R, K\rangle$.

The proof-theoretical conditions "maximal completeness" and "maximal consistency" defined below deal with variables instead of elements of a particular parameter algebra. Hence they characterize persistency of PAR with respect to all parameter algebras (Thm. 2.12).

\subsection{Definitions}

PAR is maximally complete if for all $s \in P S$ and $t \in G(S I G X)_{S} t=S P E C X t$ for some $t^{\prime} \in T$ (PSIG) (cf.2.3.). PAR is maximally consistent if for all $t, t^{\prime} \in I$ (PSIG) $t=S P E C X^{t}$ ' implies $t=P S P E C t$ '.

\subsection{Definition}

The simple reduction relation generated by $E, \vec{E}$, is the smallest relation on $T(S I G)$ resp. $Z$ (T (SIG)) such that

(i) for all $l=r$ in $E$ and $f=Z(I(S I G)) \quad n \rightarrow f r$,

(ii) for an $\sigma \in O P \sigma\left(t_{1}, \ldots, t_{1}, \ldots, t_{n}\right) \vec{E} \sigma\left(t_{1}^{\prime}, \ldots, t_{i}^{\prime}, \ldots t_{n}\right)$ if $t_{i} \vec{E}^{\prime} t_{i}^{\prime}$, 
(iii) for all $f, g \in Z(T(S I G)) ~ f \vec{E} g$ if for all $x \in X \quad f \times \vec{E} g x$.

$\vec{E}, \vec{E}$ and $\stackrel{\vec{E}}{\vec{E}}$ denote the reflexive, symmetric and reflexive-transitive closures of $\vec{E}$, respectively.

\section{12. Persistency Theorem I}

$\langle P A R, A l g(P S P E C)\rangle$ is persistent if $P A R$ is maximally complete and maximally consistent.

Proof:

"only if": By assumption, $\eta P A R\left(T_{P S I G}\right) /=P S P E C$ is an isomorphism. Since

$F_{P A R}\left(T_{P S I G}\right) /=P S P E C=G(S I G X) /=S P E C X$

$(/ 10 /, 1.6)$, we conclude

$\mathrm{T}_{\mathrm{PSIG}} /=\mathrm{PSPEC} \approx \mathrm{U}_{\mathrm{EXI}}(\mathrm{G}(\mathrm{SIGX}) /=\mathrm{SPECX})$.

The surjective resp. injective part of this isomorphism is maximal completeness resp. consistency of PAR.

The "if"-part is more tedious and given in the extended version of this paper. Its main idea is due to $H$. Ganzinger (of. $16 /$, Thm. 5).

\section{13. Corollary}

$\langle\mathrm{PAR}, \mathrm{Alg}$ (PSPEC) $\rangle$ is persistent if for all $\sigma \in O P$, sort $(\sigma) \in \mathrm{PS}$ implies $\sigma \in \mathrm{POP}$ and if for all $1=r$ in $E$ sort(l) $C$ PS implies that $1=r$ is in PE.

\subsection{Example (cf, 2.7)}

Using Corollary 2.13 we immediately observe that 〈 persistent.

\section{Extension Proofs by term rewriting}

Assuming that the "base" $\langle B P A R, K\rangle$ is persistent we now turn to tools for extension proofs which are based on the proof-theoretical characterization of conservative extensions given in the last section (2.9).

From now on we suppose that $S=B S$ and for all $A \in A l g$ (PSPEC) and $s \in S$ $G(S I G(A))_{S}$ is nonempty.

A first step towards extension criteria is the decomposition of the free SPEC (A)-congruence into simple reductions and the free BSPEC(A)-congruence:

\subsection{Definition}

Let $A \subset A{ }_{g}(P S P E C)$. A set $R$ of SIG(A)-equations is Church-Rosser w.r.t. A if for all $s \in B S$ and $t, t \in G(S I G(A))_{S}$ 


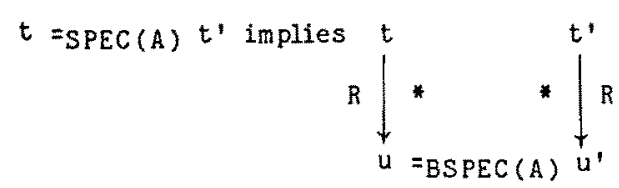

for some $u, u^{\prime} \leq G(B S I G(A))$.

\subsection{Lemma}

Suppose that for each $1=r$ in $E-B E$ op ( 1 ) contains at least one operation symbol of $O P-B O P$. For all $A \in K$ let $E(A)$ be a subset of $=B S P E C(A)$.

If $(E-B E) \cup E(A)$ is Church-Rosser w.r.t. $A$, then PAR is consistent w.r.t. $\langle B P A R, K\rangle$.

Localizing the Church-Rosser property by "confluence" and "coherence" conditions goes along with restricting equations to "normalizing" ones:

\subsection{Definitions}

Let $A$ a $A l g(P S P E C)$ and $R$ be a set of SIG(A)-equations. $t$, $G(B S I G(A))$ is an $R$-normal form of $t \in G(S I G(A))$ if $t \frac{\hbar}{R} t^{\prime}$. $R$ is normalizing w.r.t. A if for all $t$ c $G(\operatorname{SIG}(A)) t$ has an $R$-normal form. $R$ is confluent $w, r, t$. A if for all $t \in$ $G(S I G(A))$ all $R$-normal forms $t_{1}, t_{2}$ of $t$ satisfy $t_{1}=\operatorname{BSPEC}(A) t_{2}$. $\left\langle t_{1}, t_{2}\right\rangle \in$ $G(S I G(A))$ is uniformly $R$-convergent w.r.t. $A$ if some $R$-normal forms $t_{1}{ }^{\prime}, t_{2}$ ' of $t_{1}$ resp. $t_{2}$ satisfy $t_{1}:=B S P E C(A) t_{2}$, written: $t_{1} i_{R}, A t_{2}$.

$R$ is coherent w.r.t. $A$ if for all $t, t_{1}, t_{2} \in G(\operatorname{SIG}(A))$

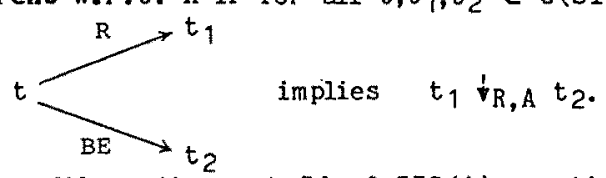

$R$ commutes with another set $R^{\prime}$ of $S I G(A)$-equations if for all $t, t_{1}, t_{2} \in G(S I G(A))$

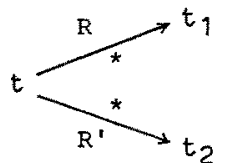

for some $t^{\prime} \in G(\operatorname{SIG}(A))$.

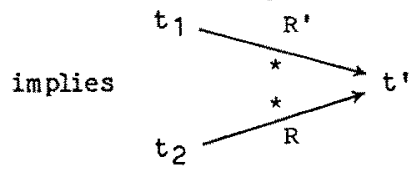

\subsection{Conpleteness Theorem I}

For all $A \subset K$ let $E(A)$ be a subset of $=S P E C(A)$. If for all $A \in K E \cup E(A)$ is normalizing w.r.t. $\langle B P A R, K\rangle$, then PAR is complete w.r.t. 〈BPAR, $K\rangle$.

Proof:

The statement immediately follows from $\mathrm{Thm}$. 2.9.1.

\subsection{Consistency Theorem I}

Suppose that for each $1=r$ in $B E \operatorname{var}(r)=\operatorname{var}(1)$ and for each $1=r$ in $E-B E I$ contains at least one operation symbol of $O P-B O P$. For all $A \subset K$ let $E(A)$ be $a$ subset of $=B S P E C(A)$. If $(E-B E) \cup E(A)$ is normalizing, confluent and coherent 
w.r.t. $A$ and commutes with $\triangle(A) \cup \triangle(A)^{-1}$ (cf. 2.8), then PAR is consistent w.r.t. 〈BPAR,K〉.

\section{Proof:}

By Lemma 3.2, it is sufficient to show that $R=(E-B E) \cup E(A)$ is Church-Rosser w.r.t. A. So let $s \in B S$ and $t, t^{\prime} \in G(S I G(A))_{s}$ such that $t=\operatorname{SPEC}(A) t^{\prime}$. There are a least number $n$ and $t_{1}, \ldots, t_{n}, u_{1}, \ldots, u_{n} \in G(S I G(A))$ with $t_{1}=t, u_{n}=t^{\prime}$ and for all $1 \leq i<n u_{i} \underset{E-B E}{E} t_{i}$ and

(i) $u_{i} \frac{E-B E}{E-1} t_{i+1}$

or (ii) $u_{i} \overrightarrow{B E} t_{i+1}$

or (iii) $t_{i+1} \overrightarrow{B E} u_{i}$

or (iv) $u_{i} \Delta(A) t_{i+1}$.

We prove $t t_{R} t$, by induction on $n$. $n=1$ implies $t \cdot \frac{*}{R} t$, and $t t_{R} t^{\prime}$ follows from normalization and confluence of $R$ w.r.t. A. Since $E(A) \subseteq=B S P E C(A)$ and for each $I=r$ in $E-B E$ op (I) $n(O P-B O P) \neq \emptyset$,

(*) for all $u \in G(B S I G(A)) u \vec{R}^{\prime} u^{\prime}$ implies $u=B S P E C(A) u^{\prime}$.

Let $n>1$. By induction hypothesis, $t_{2} t_{R}, A$ t . Hence by confluence of $R$, it remains to show $t_{1}$ in, $A t_{2}$.

The proof proceeds by deriving $t_{1} t_{R, A} t_{2}$ in each of the cases (i)-(iv) for $i=1$. ט

\section{Parameters with "built-in" logic}

From now on we deal with parameters including Boolean operators and restrict parameter algebras to those where the Boolean operators are interpreted as in propositional logic. In addition, we use if-then-else operators to simulate conditional axioms by equations.

\section{General Assumption}

Suppose that BOOL (cf. 2.7) is a subspecification of PSPEC. Moreover, let ifS be a subset of $S$ such that for all $s \in$ ifS SIG contains an operation symbol if $-s$ : bool $s \mathrm{~s} \rightarrow \mathrm{s}$ and $\mathrm{E}$ includes the equations

$$
\text { if }-s(\text { true }, x, y)=x \text { and if }-s(\text { false, } x, y)=y \text {. }
$$

Vice versa, for each $l=r$ in $E$

(i) sort(1) = bool implies 1 \& true, false \},

(ii) sort(l) $\neq$ bool implies $t \in\{$ true, false\} for all bool-sorted subterms $t$ of 1 .

\section{1. Definitions}

Let PEXT = 〈BOOL, PSPEC >. The class Log(PSPEC) is given by all PSPEC-algebras A such that $U_{P E X T}(A)$ is the Boolean algebra \{true, false\}. Hence we drop the equations true=true and false $=$ false from the equational diagram of $A$ (cf. 2.8). For all $A$ c $\log (P S P E C)$ LE(A) denotes the set of BSIG(A)-equations $1=r$ with $1 \in$ $G(\operatorname{BSIG}(A))_{\text {bool }}-\{$ true,false,$r \in\{$ true, false $\}$ and $l=\operatorname{BSPEC}(A) r$. 


\subsection{Lema}

Let $\langle B P A R, \log (P S P E C)\rangle$ be persistent. Then for all $A=\log (P S P E C)$ and $t a$ $G(B S I G(A))_{\text {bool }}$ either $t=t r$ ue or $t=$ false is in $L E(A)$.

\section{Proof:}

Let $A \subset \log (P S P E C)$. By assumption, $A=U_{B P A R} F_{B P A R}(A)$. By $T h m$. 2.8, $F_{B P A R}(A)=$ $G(B S I G(A)) /=B S P E C(A)$. Hence the statement follows from $U_{P E X T}(A)=\{$ true, false $\}$. ○

Next we define a reduction relation with conditions (contexts) in order to simulate reductions via $L E(A)$.

\subsection{Definition}

Let $L T=I(S I G)_{\text {bool }}$. The contextual reduction relation generated by $E$, $\{\stackrel{*}{E ; p}\}_{p \in L T}$, is the family of smallest relations on $T(S I G)$ such that

(i) for all $t \in T(S I G)$ and $p \in L T t \underset{E ; p}{E} t$,

(ii) for all $l=r$ in $E, f \in Z(T(S I G))$ and $p \in L T$ fl $\underset{E ; p}{*} f r$,

(iii) for all $\sigma \in O P$

$$
\sigma\left(t_{1}, \ldots, t_{i}, \ldots, t_{n}\right) \underset{E ; p}{\stackrel{*}{\longrightarrow}} \sigma\left(t_{1}, \ldots, t_{i}, \ldots, t_{n}\right)
$$

(iv) for all $s=$ ifs and $t_{1}, t_{2} \in T(S I G)_{S}$

$$
\text { if }-s\left(p, t_{1}, t_{2}\right) \underset{E ; p}{\stackrel{*}{*}} t_{1} \text { and if }-s\left(p, t_{1}, t_{2}\right) \underset{E ; \neg p}{\stackrel{*}{*}} t_{2} \text {, }
$$

(v) $t \frac{*}{E ; p \wedge q} t$ " if $t \frac{*}{E ; p} t t^{\prime}$ and $t \cdot \frac{*}{E ; q} t "$,

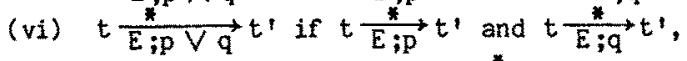

(vii) for all $t, t^{\prime} \in T(S I G) t \frac{*}{E ; \text { false }^{\prime}} t^{\prime}$.

The following lemma draws the connection between contextual and LE(A)-reductions. Contexts are now restricted to "base" terms so that contextual reductions can be regarded as "hierarchical" ones.

\subsection{Lemma}

Let $\langle B P A R, \log (P S P E C)\rangle$ be persistent. Then all $A \in \log (P S P E C), f \in Z(G(S I G(A)))$

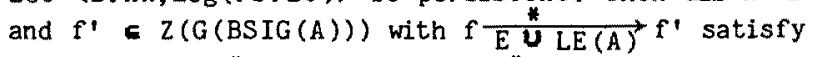

(*) ft $\underset{E \cup E(A)}{*} f t$ ' if $t \frac{*}{E ; p} t{ }^{\prime}$ and $f^{\prime} p=t r u e$ is in $L E(A)$.

Contextual reduction properties that correspond to 3.3 are defined by 4.5 and 4.11 below.

\subsection{Definition}

Let $t \in G(S I G X)$ (cf. 2.3). $t$ has contextual $E$-normal forms $t_{1}, \ldots, t_{n} \in G(B S I G X)$ if there are $n \in \mathbb{N}$ and $p_{1}, \ldots, p_{n} \in L T$ such that $p_{1} \vee \ldots \vee p_{n}=S P E C$ true and for all $i \leq i \leq n t \underset{E ; p_{i}}{\longrightarrow} t_{i}$. E is contertually normalizing if all $t \in G(S I G X)$ have contextual E-normal forms. 
To reduce normalization of $E U L E(A)$ to contextual normalization of $E$ we have to guarantee that SPEC(A) does not identify true and false:

\subsection{Definition}

PAR is logically consistent if for all $A=\log (P S P E C)$ some $B \in A$ Ig (SPEC(A)) has different interpretations of true and false.

\subsection{Lemma}

Suppose that 〈BPAR, $\operatorname{LOg}(P S P E C)\rangle$ is persistent and PAR is logically consistent. Let $A=\log (P S P E C)$. E $U \operatorname{LE}(A)$ is normalizing w.r.t. $A$ if $E$ is contextually normalizing.

\subsection{Completeness Theorem II}

Suppose that 〈BPAR, Log(PSPEC)〉 is persistent and PAR is logically consistent. If E is contextually normalizing, then PAR is complete w.r.t. 〈BPAR, Log (PSPEC) ).

Proof:

The statement immediately follows from Lemma 4.7 and Completeness Theorem 3.4.

\subsection{Example (cf. 2.7)}

Let $E=\{e 6, \ldots, e 13\}$. One easily observes that $E$ is contextually normalizing if

(*) for all $t, t^{\prime} c G(B S I G X)$ set has $(t, x), \operatorname{del}(t, x)$ and if-set $\left(x, t^{\prime} t^{\prime}\right)$ have contextual E-normal forms.

(*) follows by induction on size(t)+size(t') because we obtain

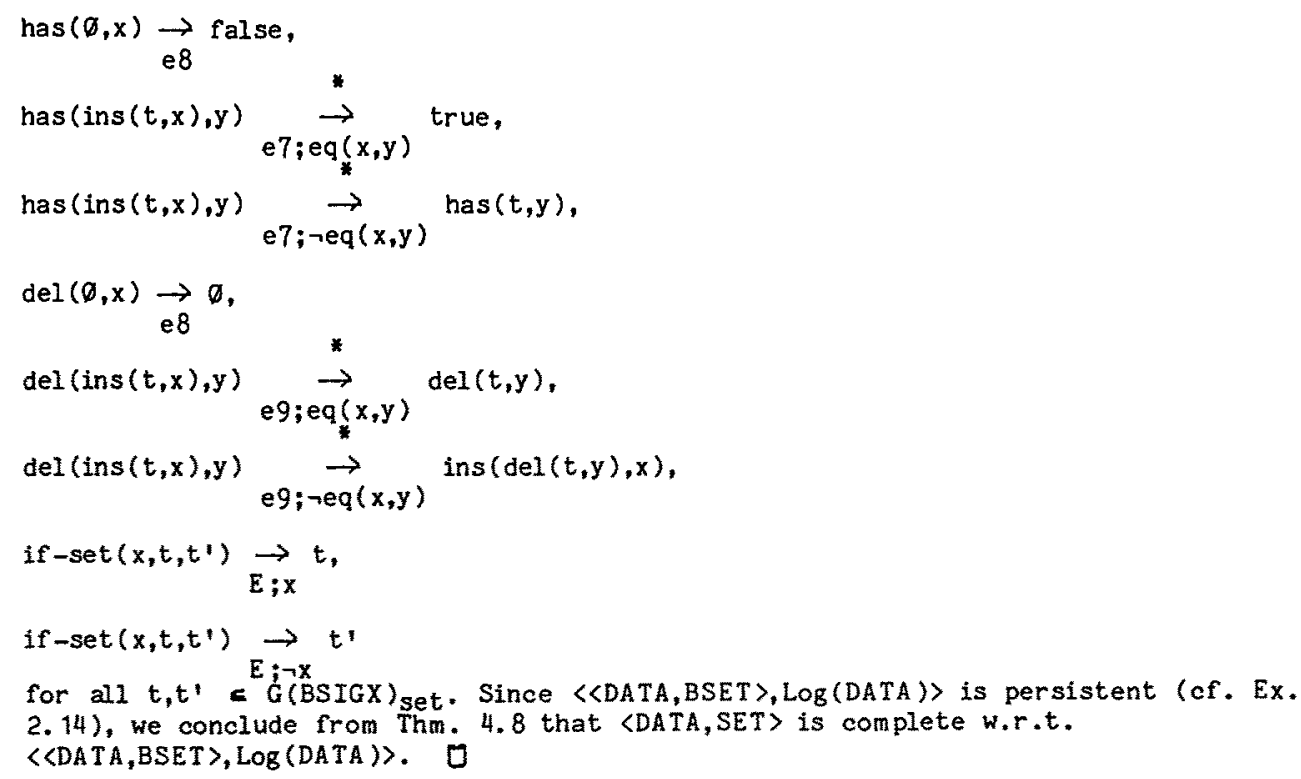


Local criteria for confluence and coherence require the "new" equations E-BE to be normalizing ( $\mathrm{cf}$. Thm. 3.5). "Base" equations (BE) are of ten not normalizing. Hence we can use Noetherian induction - to lift local criteria - only with respect to $E-B E$. But $B E$ must be considered, too. The lack of normalization of $B E$ is circumvented by working with parallel $\mathrm{BE}-$ reductions which combine independent simple reductions in one step.

\section{10. Definition}

The parallel reduction relation generated by $E, \Rightarrow$, and its reflexive closure $\stackrel{=}{\Rightarrow}$ are the smallest relations on $T(S I G)$ resp. $Z(T(S I G)$ ) such that

(i) for all $t \in T(S I G) t \underset{E}{\Rightarrow} t$,

(ii) for all $f, g \in Z(T(S I G)) \quad f \underset{E}{\Rightarrow} g$ if for all $x \in X \quad f x \stackrel{=}{\Rightarrow} g x$,

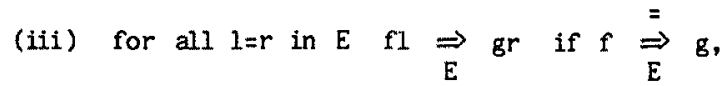

(iv) $t \underset{E}{=} t^{\prime}$ if $t \underset{E}{\Rightarrow} t^{\prime}$,

(v) for all $\sigma \in$ OP $\sigma\left(t_{1}, \ldots, t_{n}\right) \underset{E}{\Rightarrow} \sigma\left(t_{1}^{\prime}, \ldots, t_{n}^{\prime}\right)$

if $\exists 1 \leq i \leq n: t_{i} \underset{E}{\Rightarrow} t_{i}^{\prime}$ and $\forall 1 \leq i \leq n: t_{i} \underset{E}{\Rightarrow} t_{i}^{\prime}$

\section{11. Definition}

$\left\langle t_{1}, t_{2}\right\rangle=T(S I G)^{2}$ is contextually E-convergent if there are $n \in \mathbb{N}$, $p_{1}, \ldots, p_{n}, q_{1}, \ldots, q_{n} \in L T, t 1, \ldots, t_{n}^{1}, t_{1}^{2}, \ldots, t_{n}^{2} \in T(S I G)$ such that

(i) $\quad\left(p_{1} \wedge q_{1}\right) \vee \ldots \vee\left(p_{n} \wedge q_{n}\right)=$ SPEC true ,

(ii) for all $1 \leq i \leq n$

$$
\begin{aligned}
t_{1} \underset{*}{\stackrel{E ; p_{i}}{\rightarrow}} t_{i}^{1} \\
\\
t_{2} \underset{E ; q_{j}}{\rightarrow} t_{i}^{2}
\end{aligned}
$$

\section{12. Lemma}

Let 〈BPAR, Log (PSPEC)〉 be persistent, let PAR be logically consistent and $E$ be contextualiy normalizing. Let $A \in \log (P S P E C)$. If $\left\langle t_{1}, t_{2}\right\rangle \in T(S I G)$ is contextually E-convergent, then for all $f, g<Z(G(\operatorname{SIG}(A))) \quad f \stackrel{=}{\Rightarrow} g$ implies 


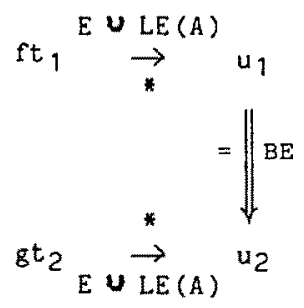

for some $u_{1}, u_{2} \in G(S I G(A))$.

\section{Critical pair conditions for consistency}

This section is the most technical one. We show that contextual convergence of certain critical pairs is sufficient for confluence, coherence and commutativity of $(E-B E) \cup L E(A)$ ( $c f .3 .3 / 5$ ). The assumptions of section 4 are still valid.

To prepare the critical pair conditions we introduce superposition relations $(5.1$ and 5.8 ) as those reductions where the lefthand side of the applied equation $1=r$ overlaps a given prefix $t$ of the term to reduce, pictorially:
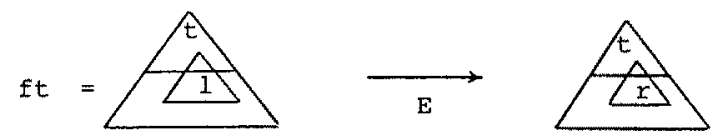

\subsection{Definition}

The simple superposition relation generated by $E$, $\{\overrightarrow{E ; f ; t}\}_{f \in Z(T(S I G)), t \in T(S I G)-X}$, is the family of smallest relations on $T(S I G)$ such that

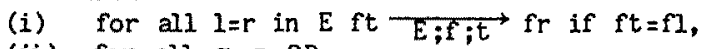

(ii) for all $\sigma \in O P$

$$
\begin{aligned}
& \text { f } \sigma\left(t_{1}, \ldots, t_{i}, \ldots, t_{n}\right) \overrightarrow{E ; f ; \sigma\left(t_{1}, \ldots, t_{i}, \ldots, t_{n}\right)} f \sigma\left(t_{1}, \ldots, t_{i}^{\prime}, \ldots, t_{n}\right) \\
& \text { if } \mathrm{ft}_{i} E_{; f ;} t_{i} f t_{i} \text {. }
\end{aligned}
$$

An instance of $5.1(i)$ is minimal if $f$ is a most general unifier of $t$ and 1 .

Let $n\left(f t \overrightarrow{E ; f ; t^{\prime}} t^{\prime}\right)$ resp. $n\left(t \vec{E} t^{\prime}\right)$ denote the least number of derivation steps 5.1(i)\&(ii) resp. 2.11(i)\&(ii) that lead to ft $\overrightarrow{E ; f ; t} t^{\prime}$ resp. $t \vec{E} t^{\prime}$.

\subsection{Proposition}

If $f t \underset{E ; f ; t}{\rightarrow} t^{\prime}$, then there are $I=r$ in $E, t_{0} \in T(S I G), t_{1} \in T(S I G)-X$ and $x \in X$ such that $\left.t=t_{0}\left[t_{1} / x\right], f t_{1}=f\right]$ and $t t^{\prime}=f[f r / x]\left(t_{0}\right), i_{.} e . I$ "overlaps" $t$ in $f t$. Proof: Straightforward induction on $n\left(f t \overrightarrow{E ; f ; t} t^{\prime}\right)$. 


\subsection{Proposition}

Let $t, t^{\prime}=T(S I G)$ and $f=Z(T(S I G))$ such that $f t \vec{E} t^{\prime}$, but not ft $\overrightarrow{E ; f^{\prime} t^{\prime}} t^{\prime}$. Then there are $x \in \operatorname{var}(t)$ and $t_{x} \in T(S I G)$ such that $f x \vec{E} t_{x}$, $n\left(\hat{f x} \vec{E} t_{x}\right) \leq n\left(f t \vec{E} t^{\prime}\right)$ and

(i) $t^{\prime}=f\left[t_{x} / x\right](t)$ if $t$ has unique variable occurrences,

(ii) $t+\stackrel{*}{\vec{E}} f\left[t_{x} / x\right](t)$ otherwise.

Proof: Straightforward induction on $n\left(f t \vec{E} t^{\prime}\right)$.

\subsection{Definition}

$E$ is linear if for each $l=r$ in $E$ each variable occurs at most once in 1.

The next lemma provides a syntactical criterion for the commutativity property in Consistency Theorem 3.5.:

\subsection{Lemma}

Suppose that 〈BPAR, $\operatorname{LOg}(P S P E C)\rangle$ is persistent, $E$ is linear and for each $I=r$ in $E I$ does not contain operation symbols of $P O P-\{t r u e, f a l s e\}$. Then for all $A$ a $\log (P S P E C) E \cup L E(A)$ commutes with $\triangle(A) \cup \triangle(A)^{-1}$.

Proof:

Let $R=E \cup \operatorname{LE}(A), R^{\prime}=\Delta(A) \cup \Delta(A)^{-1}$ and

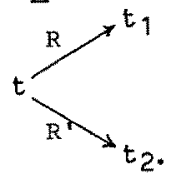

Induction on $n\left(t \vec{R} t_{1}\right)+n\left(t \overrightarrow{R^{\prime}} t_{2}\right)$ yields

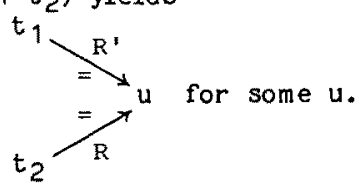

Using the superposition relation we can easily define a critical pair of $E$ into $1=r$ as a pair of

(i) the substituted righthand side fr and

(ii) the result $t$ of reducing $f l$ by some equation $I^{\prime}=r^{\prime}$ where $I^{\prime}$ overlaps 1 , pictorially:
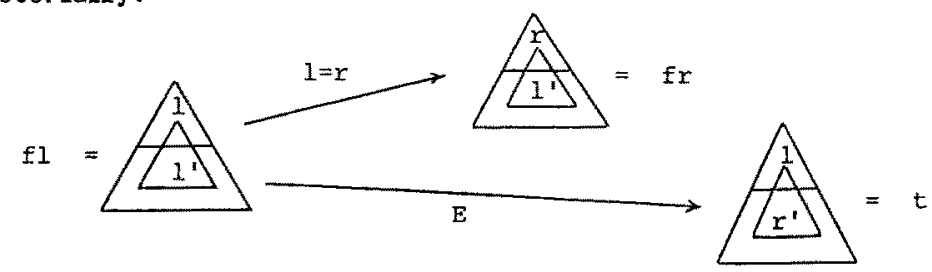

\subsection{Definition}

Let $\mathrm{fl} \underset{\mathrm{E} ; \mathrm{f} ; \mathrm{I}}{\mathrm{t}}$ and $\mathrm{l}=\mathrm{r}$ be a SIG-equation. $\langle\mathrm{fr}, t\rangle$ is called a critical pair of $E$ into $1=r$. 


\subsection{Lemma}

Let $A=\log (P S P E C)$. Suppose that for each $1=r$ in $E-B E$ op (1) contains at least one operation symbol of $O P-B O P$. Then there are no critical pairs of $E-B E$ into $L E(A)$ or of $L E(A)$ into $E-B E$. $D$

In parallel reductions we may have several equations $l_{i}=r_{i}$ applied to the same term $u$. If all outermost $l_{i}$ overlap a given prefix $t$ of $u$, we get a "superposing" parallel reduction, pictorially:
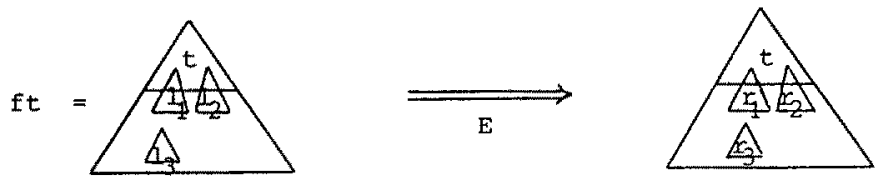

\subsection{Definition}

The parallel superposition relation generated by $E$,

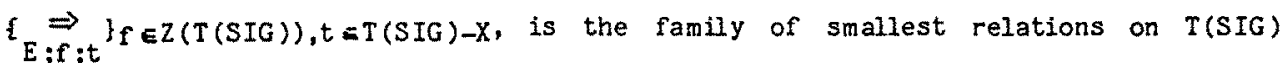
resp. $Z(T(S I G))$ such that

(i) for all $1=r$ in $E$ ft $\underset{E ; f ; t}{\Rightarrow}$ gr if $f t=n$ and $f \underset{E}{\Rightarrow} g$,

(ii) for all $\sigma \in O P f \sigma\left(t, \ldots, t_{n}\right) \underset{E ; f ; \sigma\left(t_{1}, \ldots, t_{n}\right)}{\Rightarrow} \sigma\left(t_{1}^{\prime}, \ldots, t_{n}^{\prime}\right)$

if $\exists 1 \leq i \leq n: f t_{i} \underset{E ; f ; t_{i}}{\Rightarrow} t_{i}^{\prime}$ and $\forall 1 \leq i \leq n: f t_{i} \underset{E ; f ; t_{i}}{\Rightarrow} t_{i}^{\prime}$.

Let $n\left(f t \underset{E ; f ; t}{\Rightarrow} t^{\prime}\right)$ resp. $n\left(t \underset{E}{\Rightarrow} t^{\prime}\right)$ denote the least number of derivation steps

5.8. (i) \& (ii) resp. 4.9.(i)-(v) that lead to ft $\underset{E ; f ; t}{\Rightarrow} t^{\prime}$ resp. $t \underset{E}{\Rightarrow} t^{\prime}$.

\subsection{Proposition}

If ft $\Rightarrow t^{\prime}$, then there are $t_{0}=T(S I G), g \in Z(T(S I G)), n>0$ and for all $1 \leq i \leq n{ }_{i}^{E ; f} r_{i}$ in $E, t_{i} \in T(S I G)-X$ and $x_{i} \in X$ such that $t=t_{0}\left[t_{i} / x_{i} \mid 1 \leq i \leq n\right]$, $f t_{i}=f l_{i}, f \underset{E}{\Rightarrow} g$ and $t^{\prime}=f\left[g r_{i} / x_{i} \mid 1 \leq i \leq n\right]\left(t_{0}\right), i . e .1, \ldots, I_{n}$ "overlap" $t$ in $f t$. 
Proof: Straightforward induction on $n(f t \underset{\varepsilon ; f ; t}{\Rightarrow} \underset{t}{\Rightarrow}$.).

\section{10. Proposition}

Let $t, t^{\prime} \leq T(S I G)$ and $f<Z(T(S I G))$ such that $t$ has unique variable occurrences.

ft $\underset{E}{\Rightarrow} t^{\prime}$, but not ft $\underset{E ; f ; t}{\Rightarrow} t^{\prime}$. Then there are $n>0, x_{1}, \ldots, x_{n} \in \operatorname{var}(t)$ and $t_{1}, \ldots, t_{n} \in T(S I G)$ such that $f x_{i} \underset{E}{\Rightarrow} t_{i}$ and $t^{\prime}=f\left[t_{i} / x_{i} \mid 1 \leq i \leq n\right](t)$.

Proof: Straightforward induction on $n\left(f^{t} \underset{E}{\Rightarrow} t^{\prime}\right)$.

Parallel critical pairs of $E$ into $l=r$ arise in situations like the following one where $l_{1}=r_{1}, l_{2}=r_{2}$ and $l_{3}=r_{3}$ are in $E$ :
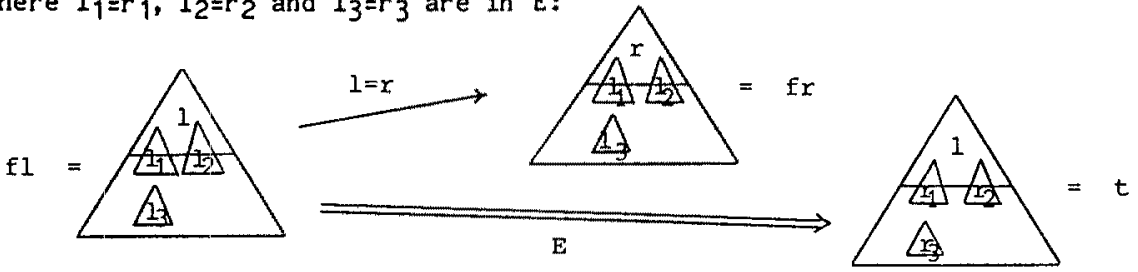

A more complicated case of a parallel overlapping can occur if $I_{1}$ shares a subterm of 1 and a prefix of $1_{3}, e .8$.

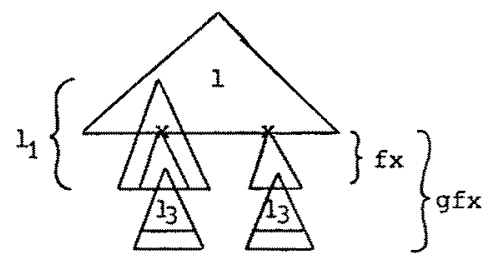

Applying $\left(l_{1}=r_{1}\right) \in E$ on one hand and $(1=r),\left(l_{3}=r_{3}\right) \in R$ on the other hand leads to a recursive critical pair of $E$ into $R$.

\subsection{Definitions}

Let $\mathrm{fl} \Rightarrow \mathrm{t}$ and $\mathrm{l}=\mathrm{r}$ be a SIG-equation. $\langle\mathrm{f} r, t\rangle$ is called a parallel critical pair of $E$ into $i f ;=r$.

Let $R$ be a set of SIG-equations and $I=r$ in $R$. Suppose that $f \overrightarrow{E_{i} f ; I} f t$ is derived from a minimal instance of 5.1(i). If for all $x \in X \mathrm{gfx}=\mathrm{hx}$ or $\mathrm{gfx} \Rightarrow \mathrm{hx}$, then 〈gft, $h r$ 〉 is a recursive critical pair of $E$ into $R$. 
$E$ is terminating if there are no infinite sequences $t_{1} \vec{E} t_{2} \vec{E} t_{3} \vec{E} \ldots$

$t$ is E-reducible if $t \longrightarrow t^{*}$ for some $t^{\prime}$.

\subsection{Critical Pair Theorem}

Suppose that 〈BPAR, $\operatorname{LOg}$ (PSPEC)〉 is persistent and PAR is logically consistent.

Let $E-B E$ be linear, terminating and contextually normalizing ( $c f .4 .5$ ), for each $l=r$ in $B E \operatorname{var}(r) \leq \operatorname{var}(1)$ and for each $l=r$ in $E-B E 1$ contains at least one operation symbol of $O P-B O P$.

Let $A=\log (P S P E C) .(E-B E) \cup L E(A)$ is confluent and coherent w.r.t. $A(c f .3 .3)$ if

(i) all critical pairs of $E-B E$ into $E-B E$,

(ii) all parallel critical pairs of $B E$ into $E-B E$,

(iii) all recursive critical pairs of $E-B E$ into $B E$

are contextually (E-BE)-convergent (cf. 4.11).

Proof: Let $R=(E-B E) \cup L E(A)$. A simple proof by Noetherian induction w.r.t. $\vec{R}$ shows that $R$ is confluent w.r.t. A if for all $t, t_{1}, t_{2} \in G(S I G(A))$

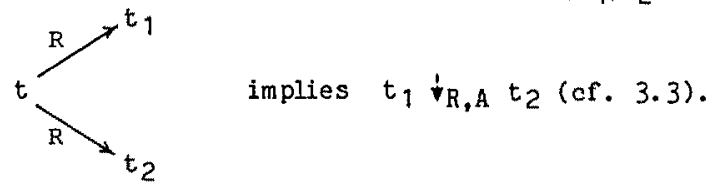

Suppose that for all $t, t_{1}, t_{2} \in G(S I G(A))$
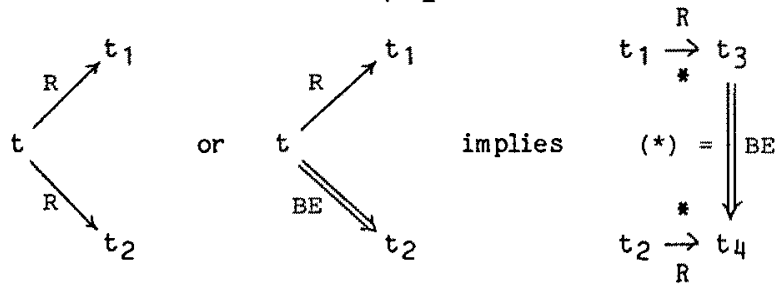

for some $t_{3}, t_{4}=G(\operatorname{SIG}(A))$. We prove by Noetherian induction w.r.t. $R$ that (2) implies (1) and coherence of $R$ w.r.t. A.

By Lemme $4.6, R$ is normalizing w.r.t. A. Hence if $t_{3}$ is not $R$-reducible, we have $t_{3}=G(B S I G(A))$ and thus $t_{4}=G(B S I G(A))$ so that $t_{1} i_{R, A} t_{2}$. If $t_{3}$ is $R$-reducible, then $t_{3} \longrightarrow t_{5}$ for some $t_{5} \in G(S I G(A))$. We obtain $t_{5} v_{R, A} t_{4}$ by induction hypothesis and thus $t_{1} r_{R, A} t_{2}$.

Hence it remains to show (2).

a) Let $t$

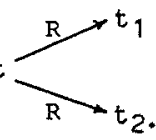

Induction on $n\left(t \rightarrow R-t_{1}\right)+n\left(t \rightarrow \vec{R} t_{2}\right)$ and a tedious case analysis leads to (*).

b)

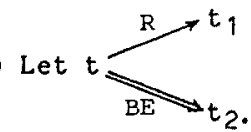


Induction on $n\left(t \vec{R} t_{1}\right)+n\left(t \Rightarrow t_{2}\right)$ and a tedious case analysis leads to (*). $\mathrm{BE}$

\subsection{Example (of. 2.7)}

Let PAR = 〈DATA,SET $\rangle$ and BPAR $=\langle$ DATA,BSET $\rangle$. One immediately verifies all assumptions of Thm. 5.12 (cf. Exs. 2.14 and 4.9) except for termination of E-BE and the critical pair conditions. For termination we refer to the recursive path ordering method (cf. $/ 5 /, / 7 /)$, which applied to $E-B E=\{(e 6), \ldots,($ e13) $\}$ provides a straightforward termination proof.

Assume that there is a critical pair of $E-B E$ into $E-B E$ or a recursive critical pair of $E-B E$ into $B E$. In both cases we would have $l=r$ in $E, f \in Z$ (T(SIG)) and $t \in$ $T$ (SIG) such that $f I \overrightarrow{E-B E ; f ; I} t$. By Prop. 5.2, there would be $l^{\prime}=r^{\prime}$ in $E-B E, t_{0} E$ $T(S I G), t_{1} \in T(S I G)-X$ and $x \in X$ such that $1=t_{0}\left[t_{1} / x\right], f t_{1}=f l^{1}$ and $t=$ $f\left[f^{\prime} / x\right]\left(t_{0}\right)$. Since for all $1=r$ in $E-B E$ op (1) $\cap(O P-B O P)=\{\operatorname{root}(1)\}$, we conclude $t_{0}=x, 1=1^{\prime}$ and $r=r^{\prime}$. Thus we have no recursive critical pair of $E-B E$ into $B E$, and if $\langle f r, t\rangle$ is a critical pair of $E-B E$ into $E-B E$, then $f r=f r^{1}=t$.

Let $\left\langle t_{1}, t_{2}\right\rangle$ be a parallel critical pair of $B E$ into $E-B E$. Then $t_{1}=f r$ and

$f 1 \Rightarrow t_{2}$ for some $1=r$ in $E-B E$ and $f \in Z(T(S I G))$. By Prop. 5.9, there are to

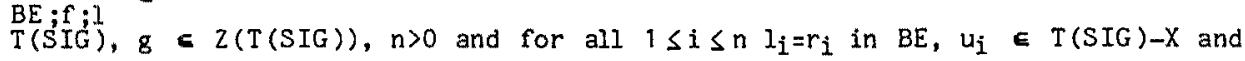

$x_{i} E x$ such that $1=t_{0}\left[u_{i} / x_{i} i 1 \leq i \leq n\right], \quad f u_{i}=f l_{i}, f \underset{B E}{\Rightarrow} g$ and $t_{2}=$ $f\left[g r_{i} / x_{i} \mid 1 \leq i \leq n\right]\left(t_{0}\right)$.

Case 1: $t_{0}=\operatorname{has}\left(x_{1}, y\right), u_{1}=\operatorname{ins}(s, x)$ and $1=r$ is $e$.

Case 1.1: $f s=$ ins $\left(f^{\prime}, f x\right)$ and $l_{1}=r_{1}$ is $e^{4}$. Then

$t_{1}=\operatorname{ifb}(e q(f x, f y)$, true has $(f s, f y))$,

$t_{2}=\operatorname{has}\left(\right.$ ins $\left.\left(85^{\prime}, g x\right), f y\right)$

so that

$t_{1}-E-B E, e q(f x, f y)$

Since

$t_{2}-E_{-B E, e q}(g x, f y)$

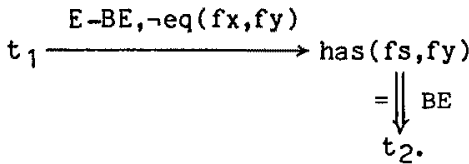

$(e q(f x, f y) \wedge e q(g x, f y)) \vee$ req $(f x, f y)$

$=B S P E C$ eq $(f x, f y) \quad V$ feq $(f x, f y)=B S P E C$ true,

$\left\langle t_{1}, t_{2}\right\rangle$ is contextually $(E-B E)$-convergent.

Case 1.2: $f s=\operatorname{ins}\left(f s^{\prime}, f x^{\prime}\right)$ and $l_{1}=r_{1}$ is e5. Then

$t_{1}=$ ifb (eq ( $\left.f x, f y\right)$, true, has (fs, fy)),

$t_{2}=\operatorname{has}\left(\operatorname{ins}\left(\operatorname{ins}\left(g s^{\prime}, g x\right), g x^{\prime}\right), f y\right)$

so that

$t_{1}-E-B E, e q(f x, f y) \vee\left(r e q(f x, f y) \wedge e q\left(f x^{\prime}, f y\right)\right)$

true

and

$t_{2}-E-B E, e q\left(g x^{\prime}, f y\right) \vee\left(n e q\left(g x^{\prime}, f y\right) \wedge e q(g x, f y)\right)$

$t_{1} \stackrel{E-B E, \text { req }(f x, f y) \wedge \text { req }\left(f x^{\prime}, f y\right)}{\longrightarrow} \operatorname{has}\left(f s^{\prime}, f y\right)$

$=\| \mathrm{BE}$

$t_{2} \underset{E-B E, \text { ᄀeq }\left(g x^{\prime}, f y\right) \wedge \text { req }(g x, f y)}{\longrightarrow} \operatorname{has}\left(g^{\prime}, f y\right)$. 
Since

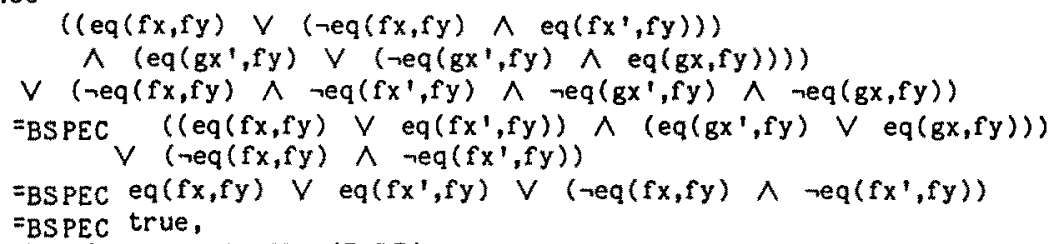

Hence all parallel critical pairs of $B E$ into $E-B E$ are contextually (E-BE)-convergent, and we conclude from Thm. 5.12 that for all $A=\log$ (PSPEC) (E-BE) $U$ LE (A) is confluent and coherent w.r.t. A.

Thms. $3.5 \& 5.12$ and Lemmata $4.7 \& 5.5$ imply the

\subsection{Consistency Theorem II}

Suppose that 〈BPAR, LOg (PSPEC) is persistent and PAR is logically consistent. Let $E-B E$ be linear, terminating and contextually normalizing, for each $1=r$ in $B E$ $\operatorname{var}(r) \varepsilon \operatorname{var}(1)$ and for each $1=r$ in $E-B E 1$ contains at least one operation symbol of $O P-B O P$, but no operation symbols of POP-\{true, false $\}$.

If all critical pairs of $E-B E$ into $E-B E$, all parallel critical pairs of $B E$ into $E-B E$ and all recursive critical pairs of $E-B E$ into $B E$ are contextually $(E-B E)$-convergent, then PAR is consistent w.r.t. 〈BPAR, Log (PSPEC)〉.

\section{15. Example (cf. 2.7)}

Let $\mathrm{PAR}=\langle\mathrm{DATA}, \mathrm{SET}\rangle$ and BPAR $=\langle\mathrm{DATA}, \mathrm{BSET}\rangle$. Using Thm. 5.14 we conclude from Ex. 5.13 that PAR is consistent w.r.t. 〈BPAR, Log(PSPEC)〉. Hence by Ex. 2.14, PAR is a conservative extension of $\langle B P A R, \log (P S P E C)\rangle$. Thus the Decomposition Lemma for Persistency (2.6) implies that $\langle P A R, \log (P S P E C)\rangle$ is persistent.

Putting together all "syntactical" criteria developed in this paper we obtain the

\section{16. Persistency Theorem II}

$\langle P A R, \log (P S P E C)\rangle$ is persistent if $P A R$ is logically consistent and contains a "base" specification BPAR such that

(i) for all $\sigma$ \& BOP sort $(\sigma)$ \& PS implies $\sigma \in$ POP,

(ii) for all $l=r$ in $B E \operatorname{var}(r)=\operatorname{var}(1)$, and $\operatorname{sort}(1) \in$ PS implies that $l=r$ is in $\mathrm{PE}$,

(iii) for all $l=r$ in $E-B E I$ contains at least one operation symbol of $O P-B O P$, but no operation symbols of POP-\{true, false\},

(iv) $E-B E$ is linear, terminating and contextually normalizing,

(v) all critical pairs of $E-B E$ into $E-B E$, all parallel critical pairs of $B E$ into $E-B E$ and all recursive critical pairs of $E-B E$ into $B E$ are contextually $(E-B E)$-convergent.

(Note also the "Boolean assumptions" at the beginning of section 4.) 


\section{References}

11 ADJ: J.A. Goguen, J.W. Thatcher, E.G. Wagner: An Initial Algebra Approach to the Specification, Correctness and Implementation of Abstract Data Types, in R.T. Yeh, ed., Current Trends in Programming Methodology, Vol. 4, Prentice-Hall (1978), 80-149

12/ ADJ: J.W. Thatcher, E.G. Wagner, J.B. Wright: Data Type Specification: Parameterization and the Power of Specification Techniques, ACM Transactions on Programming Languages and Systems 4 (1982), 711-732

13/ ADJ: H. Ehrig, H.-J. Kreowski, J.W. Thatcher, E.G. Wagner, J.B. Wright: Parameter Passing in Algebraic Specification Languages, Proc. Workshop in Program Specification, Springer LNCS 134 (1982), 322-369

14/ G. Birkhoff: On the Structure of Abstract Algebras, Proc. Cambridge Phil. Soc. 31 (1935), 433-454

15/ N. Dershowitz: Orderings for Term-Rewriting Systems, Theoretical Computer Science $17(1982), 279-301$

16/ H. Ganzinger: Parameterized Specifications: Parameter Passing and Implementation with Respect to Observability, TOPLAS 5,3 (1983), 318-354

17/ G. Huet, D.C. Oppen: Equations and Rewrite Rules: A Survey, in: R.V. Book, ed., Formal Language Theory: Perspectives and Open Problems, Academic Press (1980)

18/ J.-P. Jouannaud: Confluent and Coherent Equational Term Rewriting Systems. Application to Proofs in Abstract Data Types, Proc. Coll. on Trees in Algebra and Programming, Springer LNCS 159 (1983), 269-283

19/ P. Padawitz: Correctness, Completeness, and Consistency of Equational Data Type Specifications, Ph.D.thesis, Technische Universitat Berlin (1983)

/10/ P. Padawitz: Towards a Proof Theory of Parameterized Specifications, Proc. Symp. on Semantics of Data Types, Springer LNCS 173 (1984), 375-391

111/ H. Perdix: Proprietes Church-Rosser de Systemes de Reecriture Equationnels ayant la Propriete de Terminaison faible, Proc. Symp. on Theoretical Aspects of Computer Science, Springer LNCS 166 (1984), 97-108

/12/ J.L. Remy: Proving Conditional Identities by Equational Case Reasoning, Rewriting and Normalization, Research Report, Nancy (1983) 